In Crescendo, 2019; 10(4): 555-570

Fecha de recepción: 12/05/2019

Fecha de aceptación: 14/12/2019

\title{
ESTILOS DE APRENDIZAJE EN LOS ESTUDIANTES DE LAS LICENCIATURAS DE ADMINISTRACIÓN DE EMPRESAS Y TURISMO
}

\author{
LEARNING STYLES IN STUDENTS OF THE BACHELOR \\ OF BUSINESS ADMINISTRATION \\ AND TOURISM
}

\section{Victor G. Luna ${ }^{1}$, María M. S. Romano², Rosario Ortiz}

\section{RESUMEN}

Desde hace algún tiempo la investigación educativa explora la existencia de diversos factores relacionados con la percepción del registro y control académico mediante las calificaciones que el alumno obtiene en su aprendizaje, así como la motivación y su aprovechamiento. En este sentido la investigación permite identificar diferentes estilos de aprendizajes utilizados por los estudiantes de las licenciaturas en Administración Turística y Administración de Empresas, el propósito fue establecer una relación entre los diferentes estilos y la necesidad de generar una formación personalizada e integral. Se planteó que existen diferencias significativas entre los estilos activo y pragmático contrastados con los estilos teórico y reflexivo. El estudio se realizó en la Mixteca Poblana, México, es de tipo observacional, exploratorio y de corte transversal, aplicándose el instrumento "CHAEA". Los resultados obtenidos plantean la necesidad de profundizar en el conocimiento del alumno con respecto a su forma de pensar, de ser y de actuar para que aprenda de manera holística y significativa.

1 Doctorado en Ciencias. Profesor Investigador de Tiempo Completo. Benemérita Universidad Autónoma de Puebla, Complejo Regional Mixteca, Unidad Atlixco.vgluna@hotmail.com

2 Doctorado en Educación. Profesor Investigador de Tiempo Completo. Benemérita Universidad Autónoma de Puebla, Facultad de Administración.romanoms@hotmail.com

3 Mtra. en Administración. Profesor Investigador de Tiempo Completo. Benemérita Universidad Autónoma de Puebla, Complejo Regional Mixteca,_Unidad Atlixco.rosario_oc@hotmail.com 
"Los resultados presentados en esta investigación permitieron caracterizar el perfil de aprendizaje de los estudiantes, se puede apreciar una clara tendencia hacia el estilo de aprendizaje teórico y reflexivo por parte de las dos licenciaturas; este resultado implica para los docentes que imparten materias en ambas licenciaturas la generación de estrategias pedagógicas fundamentadas en estos estilos propios, donde los estudiantes tengan la oportunidad de pensar y responder de manera crítica y reflexiva, y la estimulación del trabajo participativo".

PALABRAS CLAVE: Empresas, turismo, aprendizaje permanente, estilos de aprendizaje.

\section{ABSTRACT}

For some time, educational research has explored the existence of various factors related to the perception of academic registration and control through the grades that the student obtains in their learning, as well as the motivation and their use. In this sense, the research carried out allows us to identify different learning styles used by undergraduate students in Tourism Administration and Business Administration, the purpose was to establish a relationship between the different styles and the need to generate personalized and comprehensive training. It was suggested that there are significant differences between the active and pragmatic styles contrasted with the theoretical and reflexive styles. The study was conducted in the Mixteca Poblana, Mexico, is observational, exploratory and cross-sectional cohort, applying the "CHAEA" instrument. The results obtained raise the need to deepen the knowledge of the student with respect to their way of thinking, being and acting so that they learn in a holistic and meaningful way.

KEY WORDS: Business, tourism, lifelong learning, learning styles.

\section{INTRODUCCIÓN}

Desde hace algún tiempo, la investigación educativa que explora el tema del logro académico o el éxito se ha extendido más allá de cuestiones "sencillas" de inteligencia y méritos académicos previos. Hay una serie de conceptos relacionados con el aprendizaje, como la percepción del control académico y la motivación del aprovechamiento, que han sido un foco de atención al intentar identificar factores que afectan el desempeño relacionado con el aprendizaje (Cassidy y Eachus, 2000). Un concepto en particular que ha proporcionado algunas ideas valiosas sobre el aprendizaje tanto en el ámbito académico como en otros es el estilo de aprendizaje. "El proceso de formación universitaria supone un constructo complejo para los diferentes actores del sistema educativo". "En el caso de directivos y profesores, cómo adaptar el currículum a las necesidades y caracterís- 
ticas de los estudiantes, sigue siendo un determinante fundamental en la construcción de procesos de enseñanza-aprendizaje de calidad (Madrid-López, et al., 2016)". "Por su parte, los estudiantes analizan y estructuran la información de diferentes formas para desarrollar el aprendizaje (Acevedo, Cavadla, y Avis, 2015)".

"De esta manera, los estilos de aprendizaje han cobrado especial importancia en la literatura científica actual relacionada con la formación inicial docente (Gil-Madrona et al., 2007; González et al., 2013)”. Actualmente existen diversos esfuerzos dirigidos a desarrollar un sistema educativo que facilite al alumno tener un aprendizaje para toda la vida, que le permita desarrollar sus capacidades de creación, innovación, producción, y su pleno desarrollo personal, con la finalidad de responder a diversas situaciones cambiantes y cada vez más complejas que existen en la sociedad.

El modelo educativo no ha dado una respuesta integral a los retos sociales contemporáneos, los fundamentos históricos y epistemológicos en los que prevalece el mecanismo, determinismo y materialismo y los valores como el control, el consumo, la competencia, la velocidad, el éxito material; se destacan en el modelo actual de educación, por ello no se ha resuelto el problema y estamos frente a un reto que sobrepasa la educación clásica (Prada, 2005), para iniciar una educación acorde con la naturaleza del ser, una formación basada en los diferentes estilos de aprendizaje que el alumno desarrolla para que "aprenda a aprender". "La aspiración de que los alumnos aprendan a aprender como requisito de una educación permanente presupone un uso eficaz y eficiente de los estilos de aprendizaje, los cuales no son a veces funcionales por ser un fenómeno complejo, en el que intervienen múltiples variables por las exigencias del contexto social con las características personales del aprendiz" (Pupo, et al., 2009).

En esta búsqueda ha tomado relevancia el concepto de estilos de aprendizaje, el cual es muy utilizado en las investigaciones psicopedagógicas actuales debido a su valor teórico y metodológico para comprender y perfeccionar el proceso de enseñanza-aprendizaje en los diferentes niveles educativos por los que transitan los alumnos. "Las tendencias pedagógicas actuales muestran un creciente interés entre los didactas y psicólogos de la educación por alejarse cada vez más de los modelos puramente instructivos para centrarse en el estudio y comprensión del propio proceso de aprendizaje". "La idea detrás de este movimiento es bien clara: cualquier intento por perfeccionar la enseñanza en aras de lograr mayor 
efectividad en la misma, tiene que transitar irremediablemente por una mejor, más clara y exhaustiva comprensión del aprendizaje, y de lo que va a ser aprendido" (Valcárcel y Verdú, 1996).

Por otra parte, Albert et al (2005) señala que estas tendencias consideran "la necesidad de tomar en cuenta el conjunto de variables individuales que inciden en el desempeño de los estudiantes (motivación, conocimientos previos, aptitudes, sistema de creencias, estilos y estrategias de aprendizaje, entre otras), las mismas que forman parte de los desafíos que enfrentan los educadores en el marco del proceso de perfeccionamiento que vive la escuela en México". Existen muchos obstáculos actualmente que detienen el proceso del desempeño de los estudiantes, uno de estos es la difícil tarea que tiene el docente para atender los diferentes estilos de aprendizaje de los alumnos, debido principalmente a que no disponen del entrenamiento adecuado con respecto al uso de estrategias de enseñanza-aprendizaje y mucho menos a un marco referencial en el cual puedan encontrar las respuestas necesarias a diversas problemáticas relacionadas con el concepto de "estilos de aprendizaje", al que los expertos de la educación atribuyen las formas particulares de comportarse de cada persona en el proceso de aprendizaje.

En este contexto, se retoma la importancia del conocimiento, la comprensión y el análisis de los estilos de aprendizaje de los futuros profesionistas, así como los estilos de enseñanza. Estudios como el de Cabrera et al., (2006), Cornejo y Redondo (2007), mencionan que es fundamental determinar las variables que se asocian con el rendimiento académico, debido a que los bajos niveles de rendimiento que muestran los alumnos universitarios están asociados con la deserción escolar y con los bajos índices de eficiencia terminal. Al respecto Edel (2003) señala que "los diferentes estilos de aprendizaje, junto a otras variables como las expectativas del alumno, el entorno personal, las habilidades sociales o el autoconcepto, se relacionan estrechamente con el rendimiento académico".

Por estos motivos, el objetivo de la investigación es realizar un análisis de las estrategias y estilos de aprendizaje que tienen los estudiantes de nuevo ingreso, generación 2017 de las licenciaturas en Administración de Empresas y de la licenciatura en Administración turística del Complejo Regional Mixteca, Unidad Atlixco. El conocimiento derivado de la exploración permitirá "adaptar los procesos de formación y alcanzar mejores resultados de aprendizaje (Catalán y 
Santelices, 2014)". La hipótesis que se planteó es que "existen diferencias estadísticamente significativas entre los estilos de aprendizaje activo y pragmático" contrastado con los estilos de aprendizaje teórico y reflexivo en los estudiantes de las dos licenciaturas.

"La palabra estilo, según la Real Academia Española (2014), proviene del latín stilus que significa carácter, peculiaridad, modo, manera o forma de hacer las cosas". "Se utiliza en la vida cotidiana para aludir a alguna cualidad distintiva y propia de una persona o grupo de personas en diferentes esferas de la actividad humana". "Al respecto Hederich y Camargo, (2000), plantean que el término refleja la necesidad de identificarse, de distinguirse entre sí, a fin de encontrar el sentido propio de identidad". "Esta es una necesidad que se manifestó desde el surgimiento de la humanidad, y por eso los romanos y griegos, de acuerdo con diferentes criterios, clasificaban a los individuos en diferentes tipologías". "Es por ello que con relación al surgimiento del término estilo de aprendizaje son muchos los investigadores que remontan su evolución histórica al estudio de caracteres relacionados con la personalidad" (Pupo, et al., 2009).

La noción de estilos de aprendizaje (o estilos cognitivos para muchos autores), tiene sus antecedentes etimológicos en el campo de la psicología. Como concepto comenzó a ser utilizado en la bibliografía especializada en los años 50 del pasado siglo por los llamados "psicólogos cognitivistas". De todos, fue H. Witkin (1954), uno de los primeros investigadores que se interesó por la problemática de los "estilos cognitivos", como expresión de las formas particulares de los individuos de percibir y procesar la información. Sus estudios y los de autores como Holzman y Clein, (1954); Eriksen, (1954); Scheerer y Goldstein (1945) "pronto encontraron eco entre los pedagogos, principalmente en países como Estados Unidos, donde ya para esta época venía generándose un amplio movimiento de reformas curriculares que clamaban por transformaciones cualitativas, la renovación de las metodologías tradicionales y el rescate del alumno como polo activo del proceso de enseñanza- aprendizaje" (Albert et al., 2005).

"Los estilos de aprendizaje pueden definirse como los diferentes mecanismos mediante los cuales la mente procesa y asimila información, influida por las percepciones, durante la adquisición de estructuras de conocimiento superior (Gil-Madrona, 2004 y Gil-Madrona et al., 2007)". "Se caracterizan por ser esquemas de comportamiento flexibles y cambiantes dependiendo, entre otros, del 
contexto del aprendiz, su experiencia, y las estrategias docentes en las que se ve inmerso (Bahamón et al., 2012; Isaza, 2014)”.

"Importantes investigaciones han demostrado que las personas tienen diferentes formas de aprender (Newble y Entwistle, 1986) y establecen distintas estrategias cognitivas, conocidas como "Estilos Cognitivos" (Cabrera y Farinas, 2001), de donde se derivan, por supuesto, los estilos de aprendizaje". "Los estilos cognitivos se definen como "la variación individual de los modos de percibir, recordar y pensar o como formas distintas de aprender, almacenar, transformar y emplear la información" (Di Bernardo, 2005:35)". "Investigadores como Newble y Entwistle, (1986) y Weinstein (1985) se refieren a los estilos de aprendizaje como un conjunto de estrategias que utilizan los sujetos de forma habitual para cumplir los objetivos de aprendizaje". "Estas estrategias abarcan no solo estrategias puramente cognitivas, sino que también involucran aptitudes motivacionales y de personalidad".

"Por tanto, los estilos de aprendizaje pueden ser considerados como los rasgos cognitivos, afectivos y fisiológicos que indican cómo los alumnos perciben, interactúan y responden a su proceso de aprendizaje dentro de un ambiente educativo (Felder, 2005)". Uno de los primeros modelos de clasificación de los estilos de aprendizaje fue el propuesto por Rita y Keneth (1993), que se centra en el componente perceptual y concreta tres estilos de aprendizaje distintos: estilo visual, estilo auditivo y estilo táctil o kinestésico. La forma en la que un individuo desarrolla su estilo de aprendizaje se ha caracterizado a través del tiempo de varias maneras y diferentes modelos teóricos (Tabla 1).

"Con respecto a la categorización de los estilos de aprendizaje, se puede mencionar la existencia de una diversidad de clasificaciones, que se han difundido por todo el mundo, sin embargo, para este estudio se tomó como referencia el modelo que plantea Honey y Mundford (1992), el cual admite diversas potencialidades de un aprendiz clasificadas en cuatro categorías fundamentales de estilos de aprendizaje (Maureira,2015)"; "permitiendo reconocer la predominancia del estilo de aprendizaje de un estudiante, y también sus características a través de estas categorías, las mismas que son consideradas fases de un proceso cíclico del aprender de todo individuo: activo, reflexivo, teórico y pragmático (Acevedo et al., 2015) (Tabla 2)”. 
"En general, los hallazgos observados en la literatura indican la predominancia de un estilo de aprendizaje más reflexivo en estudiantes universitarios (Acevedo et al., 2015). No obstante, existen estudios en los que se ha observado la prevalencia de otros estilos, caracterizados por su vinculación con procesos de enseñanza más tradicional en educación superior. A este respecto, Santizo et al. (2008) comprobaron la predominancia de un estilo de aprendizaje más teórico en estudiantes universitarios". "Por el contrario, durante los últimos años se ha observado la predominancia de estilos más propios de las nuevas corrientes de enseñanza-aprendizaje, como el pragmático, en estudiantes de diferentes carreras (Izasa, 2014), o la combinación de estilos, que son sugeridos como estilos de mayor calidad y transferencia (Bahamón et al., 2012; Cordero et al., 2015)”.

Tabla 1

TAXONOMÍA DE LOS MODELOS DE ESTILO DE APRENDIZAJE EN EL CAMPO DE LA EDUCACIÓN

\begin{tabular}{|c|c|c|c|c|c|c|c|}
\hline \multirow[b]{2}{*}{ Modelo } & \multicolumn{4}{|c|}{ Curry (1987 } & \multirow{2}{*}{$\begin{array}{c}\text { Equitación y } \\
\text { Cheema (1991) } \\
\text { Wholist analítico }\end{array}$} & \multicolumn{2}{|c|}{ Rayner y Riding ( 1997) } \\
\hline & $\begin{array}{l}\text { Preferencia } \\
\text { académica }\end{array}$ & $\begin{array}{c}\text { Interacción } \\
\text { social }\end{array}$ & $\begin{array}{c}\text { Procesamiento } \\
\text { de } \\
\text { información }\end{array}$ & $\begin{array}{c}\text { Personalidad } \\
\text { cognitiva }\end{array}$ & & $\begin{array}{l}\text { Cognitivo } \\
\text { centrado }\end{array}$ & $\begin{array}{c}\begin{array}{c}\text { Centrado en } \\
\text { el } \\
\text { aprendizaje }\end{array}\end{array}$ \\
\hline Witkin (1962) Dependencia de campo / independencia & & & & • & - & • & \\
\hline Kagan ( 1965) Impulsividad-reflexividad & & & & . & . & . & \\
\hline Holzman y Klein (1954) Nivelador-afilador & & & & . & . & . & \\
\hline Pask (1972) Equipo de publicidad & & & & . & . & . & \\
\hline Pavio ( 1971) Verbaliser-visualizador & & & & . & & - & \\
\hline Gregorc (1982) Delineador de estilos & & & & - & - & - & \\
\hline Kauffmann (1979) Asimilador-explorador & & & & - & - & - & \\
\hline Kirton ( 1994) Adaptación-innovación & & & & - & - & - & \\
\hline Allinson y Hayes ( 1996) Intuición-análisis & & & & - & . & - & \\
\hline Kolb (1984) ELM & & & - & & & & - \\
\hline Miel y Mumford (1992) LSQ & & & - & & & & - \\
\hline Vermunt (1994) LSI & & & . & & & & . \\
\hline Entwistle \& Tait (1995) Profundidad de la superficie & & & . & & & & . \\
\hline Biggs et al. (2001) SPQ & & & . & & & & . \\
\hline Schmeck et al. (1991) ILP & & & . & & & & . \\
\hline Hunt, Butler, Noy y Rosser ( 1978) Nivel conceptual & & & . & & & & . \\
\hline Dunn, Dunn y Price (1989) LSI & . & . & & & & & . \\
\hline $\begin{array}{l}\text { Reichmann y Grasha ( 1974) Estilos de aprendizaje modelo de } \\
\text { interacción } \\
\text { Ramirez y Castenada ( 1974) Formulario de calificación infantil }\end{array}$ & . & . & & . & & & $\cdot$ \\
\hline Reinert ( 1976) ELSIE & & & & . & & & . \\
\hline Hill ( 1976) Inventario de intereses de estilo cognitivo & & & & - & & & - \\
\hline Letteri (1980) Tipos de alumnos & & & & - & & & - \\
\hline Keefe y Monks ( 1986) Perfil de estilo de aprendizaje & . & . & & . & & & . \\
\hline
\end{tabular}

Fuente: Tomado y modificado de (Cassidy, 2004). 
Tabla 2

\section{ESTILOS DE APRENDIZAJE PROPUESTOS POR HONEY Y MUNDFORD}

\begin{tabular}{|c|c|}
\hline Activo-improvisador & $\begin{array}{l}\text { "Son abiertos, improvisadores y espontáneos, y no les impor- } \\
\text { ta correr riesgos o cometer errores". "Retienen mejor la infor- } \\
\text { mación haciendo algo con el conocimiento como discutirlo, } \\
\text { explicarlo o aplicarlo". "Les agrada el trabajo activo, desarro- } \\
\text { llan guías de estudio, carteleras, trabajos, talleres". }\end{array}$ \\
\hline $\begin{array}{c}\text { Teórico-metódico } \\
\text { objetivo }\end{array}$ & $\begin{array}{l}\text { "Se trata de estudiantes objetivos, con un profundo sentido } \\
\text { crítico, metódico y disciplinado, que abordan los problemas } \\
\text { desde un punto de vista lógico". "Prefieren las actividades } \\
\text { estructuradas que les permitan comprender sistemas comple- } \\
\text { jos. Prefieren las clases magistrales". }\end{array}$ \\
\hline Pragmático-realista & $\begin{array}{l}\text { "Recuerdan mejor lo que ven, figuras, demostraciones, dia- } \\
\text { gramas, imágenes". "Prefieren el apoyo de material didácti- } \\
\text { co". "Prefieren descubrir posibilidades y relaciones, les agra- } \\
\text { dan las innovaciones, captan mejor las abstracciones". "Son } \\
\text { capaces de resolver problemas rápidamente luego de captar } \\
\text { el panorama general". "Son personas realistas, directas, } \\
\text { eficaces y prácticas, prefieren planificar las acciones de mane- } \\
\text { ra que puedan ver relación entre el asunto tratado y su aplica- } \\
\text { ción". }\end{array}$ \\
\hline Reflexivo-analítico & $\begin{array}{l}\text { "Prefieren pensar detenidamente sobre el objeto de estudio y } \\
\text { trabajar solos. Aumentan la comprensión en pasos lineales, } \\
\text { pueden no entender el material, pero logran conectar lógica- } \\
\text { mente sus partes. Prefieren la elaboración de mapas concep- } \\
\text { tuales, diagramas de flujo, árboles de problemas". }\end{array}$ \\
\hline
\end{tabular}

Fuente: Correa, (2006:43). 
Los alumnos de ambas licenciaturas provienen de las regiones económicas Poblanas San Pedro Cholula y la Mixteca las mismas que contemplan una superficie de 9,341 km2, abarcando el 27\% de la superficie total del Estado de Puebla. "En esta región se desarrollan actividades como la producción vegetal, el tejido de palma, la ganadería y actualmente el cultivo del agave mezcalero, son los recursos con alto potencial económico para las familias de la región (Mora,1987; INEGI, 2000; Ibarra, 2002)".

"La actividad pecuaria en las comunidades rurales es un patrón de medida social, que tiene como principal frecuencia a la unidad de producción caprina de tipo familiar, en el sistema de economía campesina (Franco, 1999; Hernández et al., 2001), donde los riesgos en la producción pecuaria, los asume el productor y los minimiza con la producción alterna de la agricultura, comercio o con la migración a las grandes ciudades provinciales, nacionales e internacionales, de manera temporal o definitiva (Hernández-Hernández, 2006; Sánchez, 2006)”.

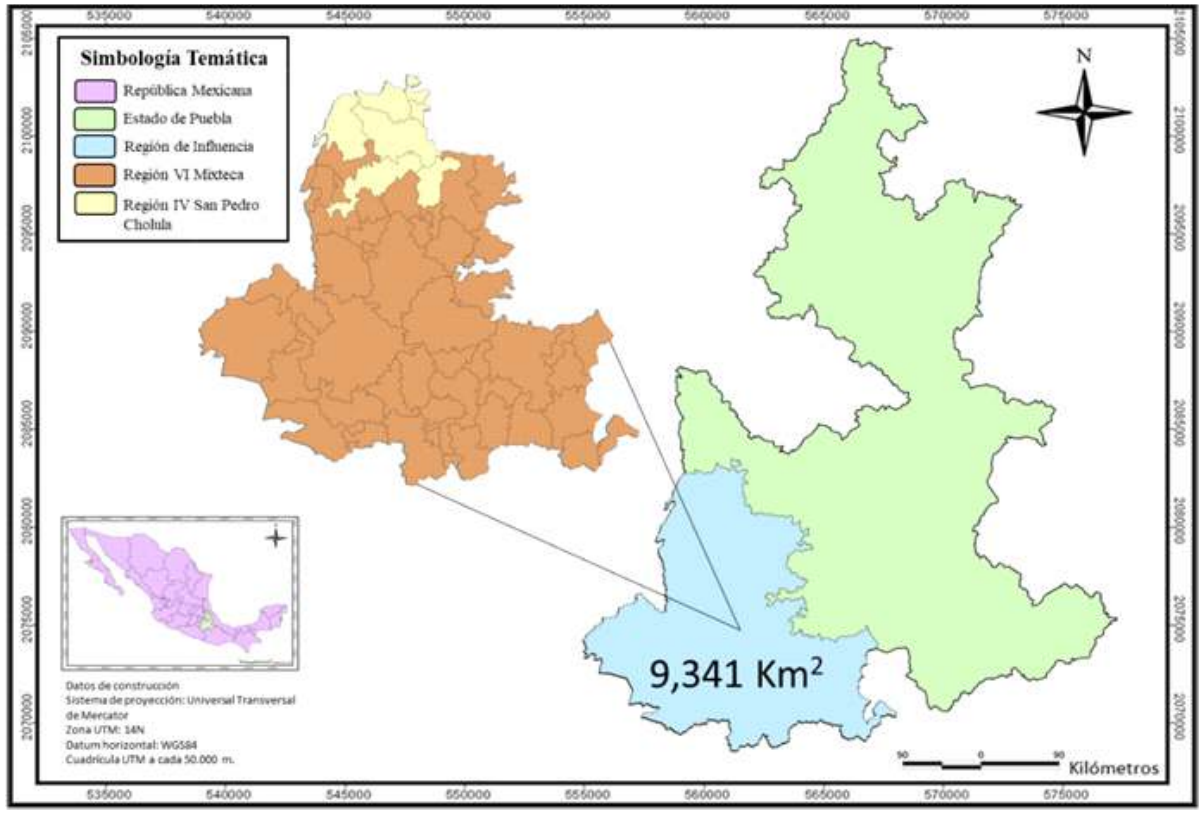

Figura1. Localización Región de Influencia San Pedro Cholula y Mixteca Poblana. 
"Las acciones agrícolas y ganaderas son el eje común de desarrollo en la mayor parte de la Mixteca Poblana; donde son indispensables para cubrir las necesidades de autoconsumo dentro de la comunidad a pesar del bajo poder adquisitivo familiar (INEGI, 2000). Los sistemas de producción se enfrentan a diferentes desafíos, destacando los cambios en los factores tecnológicos, sociales, económicos, ambientales y políticos o institucionales".

"Lo que ocasiona que buena parte de la zona en cuestión los índices de calidad de vida sean muy bajos, tanto en las oportunidades de empleo, educación, salud y vivienda, así como una limitada alimentación, falta de salarios dignos, deporte y cultura; lo cual ha ocasionado una fuerte corriente de migración de sus jóvenes a los Estados Unidos de Norteamérica (Hernández et al., 2011)”

\section{MÉTODO}

\section{Población y Muestra}

Participaron la totalidad de la generación 2017 de las Licenciaturas en Administración Turística y Administración de Empresas, pertenecientes al Complejo Regional Mixteca, Campus Atlixco de la Benemérita Universidad Autónoma de Puebla, inscritos en los cursos de Metodología de la Investigación y Administración I, respectivamente $(\mathrm{n}=64)$.

\section{Diseño y procedimiento}

Se realizó un estudio observacional, comparativo exploratorio, transversal y correlacional (Martínez, 2007). Los grupos en donde se aplicó el instrumento fueron seleccionados por conveniencia y con el consentimiento de los alumnos, los cuales accedieron de manera voluntaria a participar en dicha investigación.

Para identificar los estilos de aprendizaje se utilizó el cuestionario de HoneyAlonso "CHAEA" (Alonso y Gallegos, 2000) que consta de 80 ítems dividido en cuatro secciones de 20 ítems correspondientes a los cuatro estilos de aprendizaje (Activo, Reflexivo, Teórico y Pragmático). Se encuestaron a los alumnos en el horario y salón de clase habitual durante la segunda semana de ingreso de acuerdo con el calendario escolar 2017. Las encuestas fueron realizadas en una sola sesión con cada grupo. Primero, se entregó el cuestionario a cada alumno, posteriormente, se explicaba cómo llenarlo y por último, se dieron 10 minutos para aclarar dudas. Posteriormente, los estudiantes contestaron el cuestionario y lo entregaron al investigador al finalizar el proceso. 


\section{Variables, procedimiento de recogida y análisis de datos}

Tipos de estilos de aprendizaje. Variable discreta discontinua que representa el valor numérico de cada uno de los cuatro patrones de aprendizaje que conforman el perfil individual de cada sujeto: activo, reflexivo, teórico y pragmático. La puntuación absoluta obtenida por cada sujeto en cada grupo de veinte ítems indica el nivel que este alcanza en cada uno de los cuatro estilos. Es un instrumento con puntuación dicotómica descrita de la siguiente manera: de acuerdo (signo +), o en desacuerdo (signo -). El grado de preferencia por los estilos de aprendizaje se obtiene de la puntuación absoluta que el estudiante obtenga en cada sección. De esta forma, se obtienen los datos necesarios para valorar las preferencias de cada alumno en cada uno de los estilos y obtener, por tanto, su perfil de aprendizaje. El instrumento, permite identificar la predominancia del estilo de aprendizaje del estudiante (activo, reflexivo, teórico o pragmático) respecto a otros, o la predominancia combinada de más de un estilo de aprendizaje (Tabla 2).

Se organizaron y clasificaron los datos en función del tipo de licenciatura y se creó una base datos en el programa Excel con la información correspondiente a los datos generales (edad y género), así como los resultados obtenidos del cuestionario "CHEA". Posteriormente, se realizaron cálculos estadísticos univariantes (frecuencias y porcentajes), descriptivos de media y desviación típica de los datos mediante el uso del software IBM SPSS Statistics versión 23.

\section{RESULTADOS}

Del total de estudiantes que fueron seleccionados el $56 \%$ corresponden a la licenciatura en Administración de Empresas y 44\% corresponden a la licenciatura en Administración Turística. "La mayoría de los participantes de este estudio son mujeres" (80\%). La edad promedio fue de 21 años. El 70\% de los estudiantes manifestaron no tener experiencia laboral, frente a un 30\% que sí tenía, principalmente en actividades sin relación con su licenciatura.

"El perfil de aprendizaje de los estudiantes mostró un predominio de los estilos teórico y reflexivo". "Al comparar los perfiles de aprendizaje de las dos licenciaturas, se observó que en el estilo activo en el caso de la licenciatura en Administración de Empresas las puntuaciones iban descendiendo; mientras que en el caso de la licenciatura en Administración Turística los estilos reflexivo y activo sucedía lo contrario". 
"La prueba de homogeneidad de varianzas señaló diferencias significativas para los estilos activo p 0.045 y pragmático p 0.029 , por esta razón, se realizó la prueba ANOVA con los estilos reflexivo p 0.002 y teórico p 0.000 obteniéndose resultados estadísticamente significativos".

"Con el contraste de Scheffé y Bonferroni, se obtuvo que la media de puntuación en el estilo de aprendizaje reflexivo de los alumnos la licenciatura en Administración Turística era estadísticamente inferior (p 0.002) a la obtenida por los alumnos de la licenciatura en Administración de Empresas". "Así mismo, la media de puntuación en el estilo de aprendizaje teórico de los alumnos de la licenciatura en Administración Turística era estadísticamente inferior a la de los alumnos de la licenciatura en Administración de Empresas p 0,004 y p 0,000 respectivamente". "Para los estilos de aprendizaje activo y pragmático, no se obtuvieron diferencias significativas entre las dos licenciaturas de acuerdo con la prueba de Kruskal-Wallis".

"Con respecto a la posible relación que pudiera existir entre el estilo de aprendizaje de los estudiantes de las licenciaturas en Administración de Empresas y la licenciatura en Administración Turística con la variable de experiencia laboral, se aplicó la prueba estadística de la t de Student sin embargo, los resultados obtenidos no mostraron significancia estadística".

\section{DISCUSIÓN}

Los resultados presentados en esta investigación permitieron caracterizar el perfil de aprendizaje de los estudiantes que cursan las licenciaturas en Administración de Empresas y Administración Turística. De esta manera, se puede apreciar una clara tendencia hacia el estilo de aprendizaje teórico y reflexivo por parte de las dos licenciaturas. Este resultado implica para los docentes que imparten materias en ambas licenciaturas la generación de estrategias pedagógicas fundamentadas en estos estilos propios, donde los estudiantes tengan la oportunidad de pensar y responder de manera crítica y reflexiva, y la estimulación del trabajo participativo, mediante la asignación de tareas extra clase, acompañadas con experiencias prácticas como el desarrollo de estudios de caso, que les permita generar un razonamiento complejo a través del análisis, síntesis y autoevaluación de sus actividades de formación.

A este respecto Gordón (2016), señala que el descubrir el estilo de aprendizaje preponderante permite alcanzar aprendizajes significativos; que el estudian- 
te aprende de mejor manera cuando la actividad sugerida por su docente se identifica con su estilo de aprendizaje; que los estudiantes aprenden con más efectividad cuando existe afinidad entre el estilo de enseñanza y el estilo de aprendizaje sobresaliente, lo cual coadyuva para mejorar el desempeño de los sujetos involucrados; favorece la relación docente-estudiante; teoría y praxis; predomina una comunicación bidireccional; propicia un aprendizaje holístico.

En este sentido, el identificar el estilo de aprendizaje predominante de los alumnos de las dos licenciaturas permitirá que los docentes utilicen diversas estrategias, técnicas y herramientas para que los alumnos aprendan mejor, de manera efectiva, mejorar su desempeño, incentivar una mejor relación entre el docente y el alumno y propiciar un aprendizaje significativo de los alumnos de estas carreras. El uso de estrategias pedagógicas dentro del ámbito de la Administración ya sea con enfoque empresarial y/o turístico permitirá el desarrollo de competencias de ciencia básica como la observación, el análisis de datos, el trabajo metódico, sistematizado, organizado y objetivo.

Al respecto Alonso (2002), señala que los estudiantes universitarios tienen un estilo de aprendizaje propio y, por lo tanto, un "perfil de aprendizaje", donde se evidencia claramente su tendencia natural por alguna de las cuatro categorías definidas. "Parece ser que a medida que van desarrollando competencias en el ámbito de la propia disciplina van modificando sus preferencias, de forma que van adquiriendo más interés por la observación, el análisis de los hechos y el trabajo metódico, sistematizado y lógico; y van perdiendo el interés por aprender descubriendo, experimentando, aplicando y llevando a la práctica lo aprendido". "Coincidiendo con Aguilera y Ortiz, (2010); Castro y Guzmán, (2005), Citado por Pérez, et al. (2016), "establece que el docente universitario tenga una referencia científica de cuáles son las características que presenta el alumno con disposición a formarse como persona, como profesional y a lo largo de su vida, merece con toda justificación su conocimiento".

Por otra parte, Gordón (2016), señala que el docente debe tener presente los estilos cognitivos y de aprendizaje del alumno; sus motivaciones e intereses; comprender que los estudiantes pueden controlar sus aprendizajes; sin olvidar el aprendizaje individual y autónomo. "Por lo tanto: es necesario buscar alternativas para potenciar el desarrollo de estilos activo y pragmático en la misma medida que los estilos teórico y reflexivo, ya que de esta manera, el alumno tendría mayor facilidad para aprender de cualquier forma y aprovechar las oportunidades de aprendizaje que se encuentre a lo largo de su vida". 
Con la finalidad de disminuir el índice de deserción, el bajo aprovechamiento que se ve reflejado en las calificaciones, así como con el hecho de que el alumno solo quiera acreditar las materias sin haber aprendido y comprendido los elementos fundamentales de su carrera y, por consiguiente, tener un aprendizaje significativo que perdure durante toda su vida y le permita tener un mejor desarrollo profesional. Por su parte, el docente requiere utilizar instrumentos confiables que le permitan diagnosticar los diferentes estilos de aprendizaje que tienen sus estudiantes. A este respecto, el cuestionario de estilos de aprendizaje "CHEA" constituye una herramienta eficiente para lograr el objetivo antes mencionado, ya que nos permite conocer el estilo de aprendizaje que tiene mayormente desarrollado el estudiante.

Esta herramienta mostró que la preferencia por los estilos de aprendizaje es moderada para cada una de las cuatro escalas, con una tendencia marcada por el estilo teórico, lo que nos permite concluir que la hipótesis no se rechaza, debido a que existe una diferencia estadística significativa entre los estilos de aprendizaje activo p 0.045 y pragmático p 0.029 contrastado con los estilos de aprendizaje reflexivo p 0.002 y teórico p 0.000 . Es importante señalar que las estrategias de enseñanza que pudieran utilizar los docentes de acuerdo con los estilos de aprendizaje identificados en los alumnos, muchas veces quedan condicionadas por la parte hermética del sistema universitario que de modo parcial proporciona determinados campos de aprendizaje basados únicamente en lo teórico y lo reflexivo, que se encuentran dados de facto por el sistema y que impiden o dificultan en gran medida el desarrollo del estilo de aprendizaje activo y pragmático, limitando con ello la creatividad del docente. Por lo que, no sorprende que la práctica docente se encuentre enfocada solamente en la enseñanza teórica y poco o nada aplicada y reflexiva.

La caracterización de los estilos de aprendizaje que tienen los alumnos de las licenciaturas en Administración Turística y la licenciatura en Administración de Empresas del Complejo Regional Mixteca, Unidad Atlixco se ha convertido en un primer paso para el proceso de personalización del aprendizaje del alumno en la unidad académica: destacando los siguientes beneficios:

1) La identificación, análisis y significado que se realiza en esta investigación con respecto al estudio de los estilos de aprendizaje, justifica la necesidad de profundizar mediante la observación y el diálogo entre el docente y el alumno las diferentes formas en que aprende, así como a la utilización de estrategias didácticas que se encuentren bien planeadas, que existan condiciones de lugar y espacio en donde converjan estilos y actividades diversas de aprendizaje. 
2) La importancia en que los docentes conozcan la forma de ser, de pensar y de actuar de los alumnos permitirá planear y utilizar distintos métodos, técnicas, procedimientos e instrumentos de evaluación que puedan generar un aprendizaje significativo y para toda la vida en el alumno.

Los ámbitos actuales de los programas de estudios universitarios del Complejo Regional Mixteca, Campus Atlixco de la Benemérita Universidad Autónoma de Puebla, ofrecen amplias posibilidades en modalidades de enseñanza, sin embargo, su diseño y líneas de acción sobre todo en la parte operativa por parte de los profesores, convierten los escenarios de enseñanza-aprendizaje en sistemas rígidos, cerrados y extraordinariamente estructurados que en muchas ocasiones no han sido lo suficientemente desarrollados por los profesores.

\section{REFERENCIAS BIBLIOGRÁFICAS}

CastrAcevedo, D., Cavadla, S., y Alvis, A. (2015). Estilos de Aprendizaje de los Estudiantes de la Facultad de Ingeniería de la Universidad de Cartagena (Colombia). Formación Universitaria, $8(4), 15-22$.

Aguilera, E. y Ortiz, E. (2010). La caracterización de perfiles de estilos de aprendizaje en la educación superior, una visión integradora. Revista Estilos de Aprendizaje.5 (5). 26-41. Recuperado de: http://earningstyles.uvu.edu/index.php/jls/article/view/117/80

Bahamón, M., Vianchá, M., Alarcón, L., y Bohórquez, C. (2012). Estilos y estrategias de aprendizaje relacionados con el logro académico en estudiantes universitarios. Pensamiento Psicológico, $9(1), 115-12$.

Cabrera, Lidia, Bethencourt, José Tomás, Álvarez Pérez, Pedro y González Afonso, Míriam (2006, noviembre 8). El problema del abandono de los estudios universitarios [Text.Article]. Recuperado el 26 de julio de 2017, a partir de http://www.uv.es/RELIEVE/v12n2/RELIEVEv12n2_1.htm

Catalán, X., y Santelices, M. (2014). Rendimiento Académico de estudiantes de distinto nivel socioeconómico en universidades: El caso de la Pontificia Universidad Católica de Chile. Calidad de Educación, 3, 22-52.

Cordero, E., Lizano, C., Ortiz, A., y Arias, F. (2015). Relationship between learning style and academic performance in students of the pharmacy program at Universidad de Costa Rica. Revista Digital de Investigación en Docencia Universitaria, 0 (2), 49-63.

Diccionario de la lengua española. (2014). Edición del Tricentenario. Recuperado el 26 de julio de 2017, a partir de http://dle.rae.es/?id=GsFwSJ6

Gordón, F. D. R. A. (2016). Implicaciones del conocimiento de la teoría de los estilos de aprendizaje en el ejercicio profesional del docente universitario. Journal of Learning Styles, 9(18). 
Victor G. Luna, María M. S. Romano, Rosario Ortiz

Hernández, Z.J.S., Rodero, E., Herrera, M., Delgado, J.V., Barba, C. y Sierra, A. (2001). La Caprinocultura en la Mixteca Poblana (México). Descripción e identificación de factores limitantes. Arch.Zootec.,50: 231-239.

Holzman, P. S., y Klein, G. S. (1954). Cognitive System-Principles of Leveling and Sharpening: Individual Differences in Assimilation Effects in Visual Time-Error. The Journal of Psychology, 37(1), 105-122. https://doi.org/10.1080/00223980.1954.9916135

Honey, P., y Mumford, A. (1992). The manual of learning styles, 3rd. Maidenhead: Peter Honey.

Madrid-López, P.D., Prieto-Ayuso, A., Samalot-Rivera, A., y GilMadrona, P. (2016). Evaluación de una propuesta extraescolar de conductas apropiadas en educación física y deportiva. Retos. Nuevas tendencias en Educación Física, Deporte y Recreación 30, 36- 42.

Maureira, F. (2015). CHAEA-36: Adaptación del cuestionario Honey-Alonso de estilos de aprendizajes para estudiantes de educación física de Chile. Revista Electrónica de Psicología Iztacala, 18(3), 1133-1152.

Ortiz, A., y Canto, P. (2013). Estilos de aprendizaje y rendimiento académico en estudiantes de ingeniería en México. Revista de estilos de aprendizaje, 6(11), 160-177.

Pérez, J. C. V., Serrano, R. M., Recio, G. M., y López, L. V. (2016). Las estrategias de enseñanza como factor de cambio en los estilos de aprendizaje. Un estudio longitudinal. Journal of Learning Styles, 9(18).

Prada Madrid, E. (2005). Las redes de conocimiento y las organizaciones. Revista Bibliotecas y Tecnologías de la información, 2(4), 16-25.

Sánchez, T.Y. (2006). Diagnóstico productivo para sustentar las unidades de producción familiar caprinas en la Mixteca Poblana: Tehuaxtla y Maninalcingo. Tesis de Licenciatura. EMVZ BUAP. Tecamachalco. Puebla. México.

Santizo, J., García, L., y Gallego, D. (2008). Dos métodos para la identificación de diferencias de estilos de aprendizaje entre estudios donde se ha aplicado el CHAEA. Revista Estilos de Aprendizaje, 1(1), 28-42. 